Research Article

\title{
Thermometric Titration for Rapid Determination of Trace Water in Jet Fuel
}

\author{
Jian-Qiang Hu, ${ }^{1}$ Jian-Jian Zhang, ${ }^{1}$ Shi-Zhao Yang, ${ }^{1}$ Yong-Liang Xin, ${ }^{1}$ Li Guo, ${ }^{1}$ and \\ Ting Yao ${ }^{1,2}$ \\ ${ }^{1}$ Department of Aviation Oil and Material, Air Force Logistics College, Xuzhou 221000, China \\ ${ }^{2}$ Analysis and Testing Center, Huangshan University, Huangshan 245041, China
}

Correspondence should be addressed to Jian-Qiang Hu; hjq555918@sohu.com and Ting Yao; ttyaoting@163.com

Received 17 July 2017; Accepted 11 September 2017; Published 30 October 2017

Academic Editor: Binoy K. Saikia

Copyright (C) 2017 Jian-Qiang Hu et al. This is an open access article distributed under the Creative Commons Attribution License, which permits unrestricted use, distribution, and reproduction in any medium, provided the original work is properly cited.

\begin{abstract}
Water content in jet fuels is detected by thermometric titration (TMT), and the optimal detected system is 2,2-dimethoxypropane as titrant, cyclohexane and isopropanol as titration solvents, and methanesulfonic acid as catalyst in this method. The amounts of oil, concentration and delivery rate of titrant, volumes, and the reliability and accuracy of thermometric titration were emphasized. The results show that the accuracy, validity, and reliability of TMT are excellent by different indicated spiked water contents. The obtained results between TMT and Karl Fischer titration have been proven to be in accord. But, the duration of titration merely spends $3-5 \mathrm{~min}$ in the whole process, greatly shortening the detected time. Therefore, rapid and accurate determination of trace water in a jet fuel can be realized by TMT.
\end{abstract}

\section{Introduction}

Water in jet fuels can result in fuel system icing, microbial contamination, and corrosion, which was considered as an important quality of fuel performance. Under a lowtemperature environment, dissolved water content of jet fuels is reduced and free water in fuels generates small ice crystals that block the oil line and decrease the normal fuel supply, so as to threaten flight safety [1]. Thus, water content in jet fuels must be strictly controlled.

At present, several field and laboratory methods are used to determine water in oil products. Field method is mainly visual observation method (VOM), which is conducted for the qualitative determination of water. However, as is characteristic of poor accuracy and repeatability, VOM cannot perform accurate quantitative determination of water in oil products [2]. Laboratory methods mainly include sensor method, solvent reflux, and Karl Fischer titration. Sensor method can achieve online measurement, but which requires established standard curves for each oil product.
Meanwhile, the results are bound to be affected by the environment [3]. Solvent reflux method can directly give free water contents in oil products; however, the method in obtaining data is subjective, complicated, time-consuming, and low accuracy [4]. Although the Karl Fischer method can obtain accurate water contents, because of highly toxic reagents, complicated preparation, and short service life, it has seriously hindered the practical uses [5]. Therefore, thermometric titration (TMT) is presented and attracted more and more attention due to rapidity, convenience, automation, and particularly excellent accuracy and applicability. TMT has been successfully applied in product quality, process control, and analysis of acidic substances and ions in aqueous and nonaqueous phase systems of different areas, such as petroleum, chemical engineering, electric power, pharmacy, and hygiene [6-11].

In the present investigation, the water of jet fuel was subjected to determination by TMT. As the same time, results by TMT are compared with by Karl Fischer, and accuracy and repeatability of results by TMT are further testified. 


\section{Experiments}

2.1. Experimental Principle. TMT is an analytical method used to determine the content of substances, which is measured on the basis of different values of enthalpy of reaction [12]. Water content through TMT is based on the endothermic reaction between 2,2-dimethoxypropane (DMP) and water. Water content in jet fuels can be calculated when the inflection point in temperature-titration volume curves is used as titer of the titrant.

Water content in jet fuel is detected with DMP as titrant. DMP has undergone an endothermic reaction with water under the catalytic action of acids, and its enthalpy is $+27.6 \mathrm{~kJ} / \mathrm{mol}$. The reaction equation of DMP and $\mathrm{H} 2 \mathrm{O}$ is exhibited as (1).

$$
\mathrm{CH}_{3} \mathrm{C}\left(\mathrm{CH}_{3} \mathrm{OH}_{2}\right)_{2} \mathrm{CH}_{3}+\mathrm{H}_{2} \mathrm{O} \stackrel{\mathrm{H}+}{\rightarrow} 2 \mathrm{CH}_{3} \mathrm{OH}+\left(\mathrm{CH}_{3}\right)_{2} \mathrm{CO} \text {. }
$$

2.2. Reagent and Instrument. DMP (98\%), isopropyl alcohol (A.R.), cyclohexane (A.R.), and methanesulfonic acid (MSA; 99\%) were purchased from the Shanghai China National Medicines Corporation Ltd. Number 3 jet fuel was purchased from the Tianjin branch of Sinopec. The obtained reactants were dehydrated through a molecular sieve and placed in a dryer.

A self-developed automatic TMT device was equipped with titration software, high-precision temperature-sensing probe, volumetric dispenser, and magnetic stirrer. The titration software has the unique capability of processing large number of data points at the rate of 8 measurements every second which is critical for reliable detection of the endpoint; the probe can measure the temperature to $0.01^{\circ} \mathrm{C}$ and have a response time of less than $0.3 \mathrm{~s}$; volumetric dispenser can consistently deliver desired volume of titration solvent. Electronic scales from German Sartorius Corporation are capable of weighing to $\pm 0.1 \mathrm{mg}$. $10 \mu \mathrm{L}$ and $1000 \mu \mathrm{L}$ microinjectors were purchased from Shanghai Anting Microsyringe Plant. The SC-3 Karl Fischer titrator was produced by Shandong Zhonghui Instrument Co. Ltd.

\subsection{Reagent Preparation}

(1) Preparation of the titrant: $12.5 \mathrm{~mL}$ DMP was accurately weighed, added into $100 \mathrm{~mL}$ volumetric flask, and dissolved into a constant volume with cyclohexane; the DMP titrant with a concentration of approximately $1.0 \mathrm{~mol} / \mathrm{L}$ was obtained. Then, $0.1 \mathrm{~mol} / \mathrm{L}$, $0.5 \mathrm{~mol} / \mathrm{L}, 1.5 \mathrm{~mol} / \mathrm{L}$, and $2.0 \mathrm{~mol} / \mathrm{L} \mathrm{DMP}$ titrants were prepared according to the above method.

(2) Preparation of the titration solvent: the titration solvent was obtained by mixing isopropyl alcohol and cyclohexane with a volume ratio of $2: 3$.

(3) Preparation of the standard aqueous solution: $1 \mu \mathrm{L}$, $2 \mu \mathrm{L}, 4 \mu \mathrm{L}, 8 \mu \mathrm{L}, 16 \mu \mathrm{L}$, and $20 \mu \mathrm{L}$ distilled water were dissolved into $10 \mathrm{~g}$ anhydrous isopropyl alcohol, and standard solutions with mass fractions of $0.01 \%$ $(w / w), 0.02 \%(w / w), 0.04 \%(w / w), 0.08 \%(w / w)$
TABLE 1: Results of different mass.

\begin{tabular}{lcccccc}
\hline Mass (g) & 2.0 & 5.0 & 10.0 & 15.0 & 20.0 & 30.0 \\
Recovery rate (\%) & 97.2 & 99.3 & 100.8 & 102.1 & 104.8 & 105.5 \\
\hline
\end{tabular}

$0.16 \%(w / w)$, and $0.2 \%(w / w)$ were obtained, which was used to calibrate the concentration of DMP titrant.

\subsection{Experimental Method}

(1) TMT: the appropriate amount of jet fuel was accurately weighed and added to the thermal insulation reaction flask; then, $20 \mathrm{~mL}$ titration solvent and $200 \mu \mathrm{L}$ MSA were added to the flask and fully dissolved with the stirring speed of $200 \mathrm{r} / \mathrm{min}$; the thermistor and titrant delivery line were placed into the sample. DMP titrant with appropriate concentration was delivered to the sample at a constant speed. The TMT software was used to record the temperature and titrant volume of the titration system; there was a change in the slope of the plot of temperature versus volume, which indicated the endpoint of the titration. Endpoints were determined by calculating the second derivative and facilitating the determination of the endpoint. During titration, there is a blank to be determined. The blank titration is a summation of all delays inherent in the titration solution under defined experimental conditions. These delays were caused from kinetics of the chemical reaction between titrant and titrand, sensor response, mixing inefficiencies, electronic transfer, and computation of data. The volumes of the titrant obtained in the blank titration were subtracted from those in the initial titration data of fuel samples. Each titration experiment was performed three times.

(2) Karl Fischer titration: the water content in jet fuel was measured by Karl Fischer method of ASTM D6304.

\section{Results and Discussion}

3.1. Effect of the Mass of Fuel, Concentration, and Delivery Rate of Titrant on Thermometric Titration. The water contents in jet fuel are relatively small, so the mass of fuel, concentration, and delivery rate of titrants will affect the accuracy of the determination results in thermometric titrations. As Table 1 shows, the mass was $2.0 \mathrm{~g}$ and there is a slight deviation. The mass was between $5.0 \mathrm{~g}$ and $15.0 \mathrm{~g}$ and the test value was basically consistent with the real value. The mass reached or exceeded $20.0 \mathrm{~g}$ and there was a large deviation. These observations show that the effects of sample mass on the determination results are relatively small in a reasonable range.

The results of titrant consumption under different concentrations of titrants are listed in Table 2, from which we can see clearly the concentrations of the titrant have great influence on determination result of TMT. The smaller the concentration of the titrant is, the larger the consumption 
TABLE 2: Results of different concentrations of titrant.

\begin{tabular}{lccccc}
\hline Concentration $\left(\mathrm{mol} \cdot \mathrm{L}^{-1}\right)$ & 0.1 & 0.5 & 1.0 & 1.5 & 2.0 \\
Recovery rate $(\%)$ & 108.2 & 101.1 & 99.5 & 98.3 & 92.2 \\
\hline
\end{tabular}

TABLE 3: Results of different delivery rates of titrant.

\begin{tabular}{lcccccc}
\hline Delivery rate $\left(\mathrm{mL} \cdot \mathrm{min}^{-1}\right)$ & 0.5 & 0.8 & 1.0 & 1.5 & 2.0 & 3.0 \\
Recovery rate $(\%)$ & 105.8 & 101.5 & 99.3 & 96.5 & 92.8 & 89.5 \\
\hline
\end{tabular}

TABLE 4: Corresponding relationship between spiked water content and DMP titer.

\begin{tabular}{lccccc}
\hline Spiked water content (\%) & 0.01 & 0.02 & 0.03 & 0.04 & 0.05 \\
DMP titer $(\mathrm{mL})$ & 0.947 & 1.013 & 1.073 & 1.127 & 1.173 \\
\hline
\end{tabular}

of the titrant volume is, which results in larger deviation. The higher the concentration of the titrant is, the smaller the consumption of the titrant volume is, which results in larger error on determination results. In summary, the most optimal titrant concentration is between $0.5 \mathrm{~mol} / \mathrm{L}$ and $1.5 \mathrm{~mol} / \mathrm{L}$. In addition, in order to compare Karl Fisher conveniently, the concentration of titrant was used as $1.0 \mathrm{~mol} / \mathrm{L}$ in all experiments.

As showed in Table 3, when the delivery rate of the titrant was $0.5 \mathrm{~mL} \cdot \mathrm{min}^{-1}$, the recovery rate was $105.8 \%$. When the delivery rate was between $0.8 \mathrm{~mL} \cdot \mathrm{min}^{-1}$ and $1.0 \mathrm{~mL} \cdot \mathrm{min}^{-1}$, the test value and real value of titrant consumption were quite close. When the delivery rate reached or exceeded $1.5 \mathrm{~mL} \cdot \mathrm{min}^{-1}$, deviation is large. Therefore, the delivery rate of titrant played an important role on the determination results for TMT, and the most optimal titration rate is between $0.8 \mathrm{~mL} \cdot \mathrm{min}^{-1}$ and $1.0 \mathrm{~mL} \cdot \mathrm{min}^{-1}$.

3.2. Determination of the Standard Water Content. The prepared DMP titrant was calibrated by five groups of standard solutions. Linear fitting of the data points was conducted, and titrant concentration was calculated according to the slope. The determination results were displayed in Table 4.

Linear fitting of the standard water content and DMP titer was conducted. As demonstrated in Figure 1, the calibration curve of DMP was $y=5.6650 x+0.8967$. According to the calculation based on the linear fitting equation, the slope of the fitted straight line was $k=5.6650$. The straight slope $k$ was the value in millimeters of consumed titrant per $1 \%$ water content in $10 \mathrm{~g}$ jet fuel. According to the chemical equation of DMP and $\mathrm{H}_{2} \mathrm{O}$, the ratio between DMP and $\mathrm{H}_{2} \mathrm{O}$ with regard to the stoichiometric number of the chemical reaction was $1: 1.0 .9807 \mathrm{~mol} / \mathrm{L}$ DMP was be obtained according to the experimental results.

Jet fuel with mass of $10.015 \mathrm{~g}$ was accurately weighed. Then, spiked water with different mass fractions was added to the jet fuel and mixed with $20 \mathrm{~mL}$ titrant and $200 \mu \mathrm{L}$ MSA. Stirring velocity was set at $200 \mathrm{r} / \mathrm{min}$, and $1.0 \mathrm{~mol} / \mathrm{L}$ titrant was used at a titration velocity of $0.8 \mathrm{~mL} / \mathrm{min}$. As

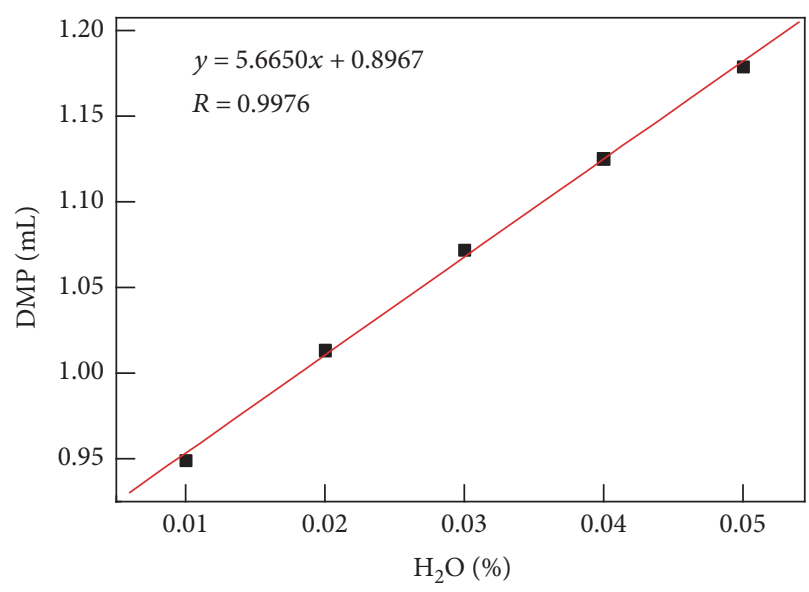

FIgURE 1: Calibration curve of the DMP.

TABLE 5: Results of jet fuels with different standard water contents.

\begin{tabular}{lcccccc}
\hline Spiked water content (\%) & 0.01 & 0.02 & 0.04 & 0.08 & 0.16 & 0.20 \\
$\begin{array}{l}\text { Spiked recovery rate (\%) } \\
\begin{array}{l}\text { Relative standard } \\
\text { deviation (\%) }\end{array}\end{array}$ & 102.4 & 101.5 & 98.6 & 99.0 & 101.5 & 98.2 \\
\hline
\end{tabular}

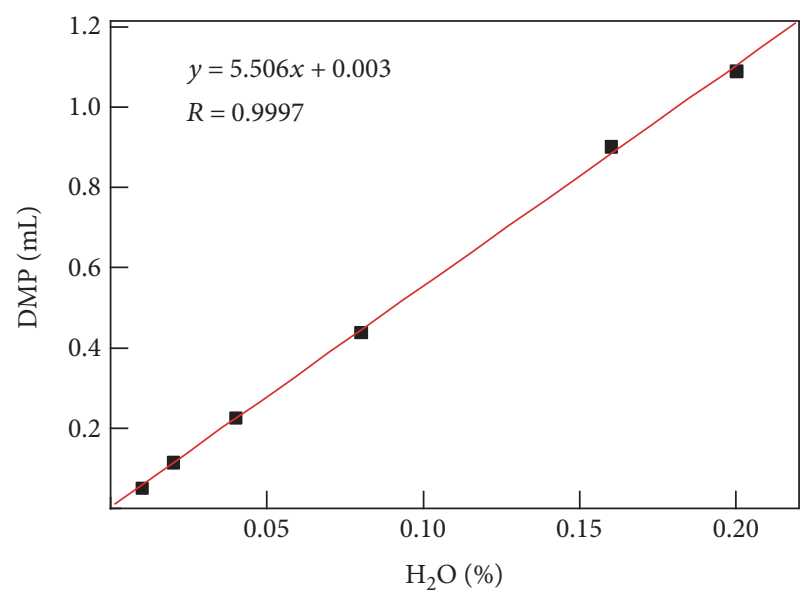

FIGURE 2: Linear regression equation of determining the spiked water contents.

shown in Table 5, the spiked recovery rates under highand low-water contents were high, that is, they are within $98 \%$ to $103 \%$

Linear fitting of the standard water content and DMP titer was established. Figure 2 demonstrated the linear regression equation between the spiked water contents and DMP titers. The linear regression coefficient $R$ was 0.9997 . When the spiked water contents within $0.01 \%(\mathrm{~m} / \mathrm{m})$ and $0.2 \%$ $(\mathrm{m} / \mathrm{m})$ were tested in the jet fuels, the determination results were highly accurate with favorable repeatability.

3.3. Determination of the Water Content in Jet Fuels. Under room temperature $\left(20^{\circ} \mathrm{C}\right)$ and humidity $(80 \%)$ conditions, 


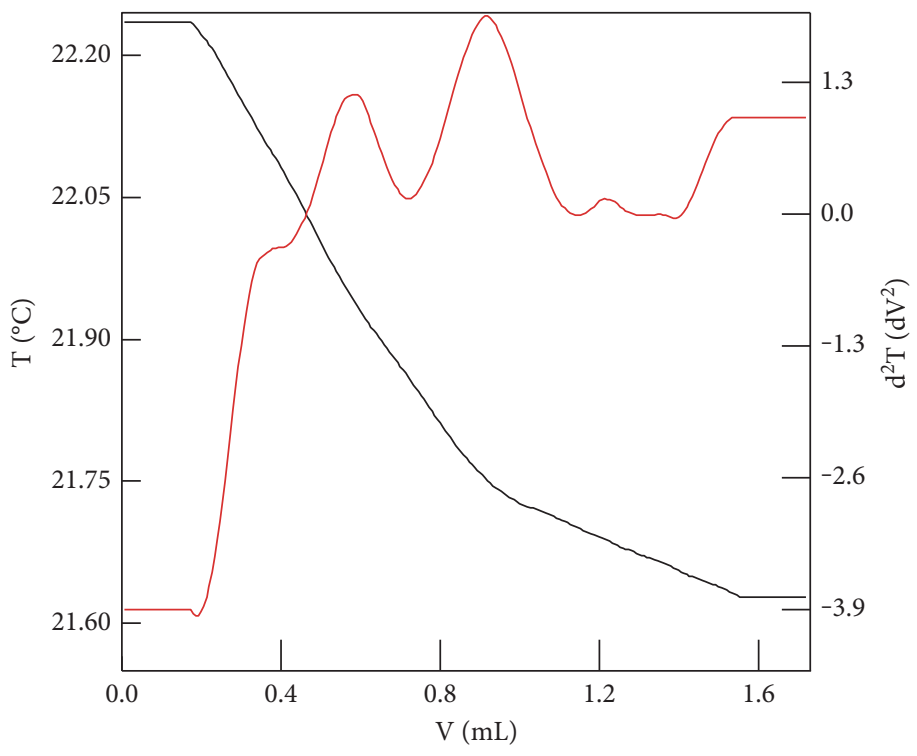

FIgURE 3: The curves of temperature titration.

jet fuels with mass of $10 \mathrm{~g}$ were accurately weighed. Calibrated DMP was used to determine the water contents in jet fuels. The curve of temperature titration was shown in Figure 3.

The selection of the endpoint of titration was automatically carried out with no interference with the manual. A second derivative was rapidly formulated to confirm the automatic selection of endpoint by titration software. TMT and Karl Fischer method were used to determine the water content in jet fuels under different conditions. As displayed in Table 6, at different temperatures, the water determination results obtained through TMT were accurate. Thus, this method was appropriate for determining water content in jet fuels.

3.4. Mechanism Analysis of TMT Determining Water. On the basis of the influence of the laws of the titration conditions, the reaction principle between DMP and $\mathrm{H}_{2} \mathrm{O}$ was analyzed during the determination process. The DMP titrant contained $\mathrm{H}_{3} \mathrm{C}-\mathrm{OH}$ groups, which would react with water by absorbing heat and realizing water determination. The reaction mechanism between DMP and $\mathrm{H}_{2} \mathrm{O}$ was shown in Figure 4.

The structure of DMP titrant contains two methoxy groups connected to one carbon atom forming large sterically hindered and strong chemical activity. This structure easily undergoes nucleophilic substitution with water. Under catalyst-free conditions, $\mathrm{S}_{\mathrm{N}} 2$ nucleophilic substitution occurs in the reaction. Particularly, $\mathrm{H}_{2} \mathrm{O}$ induces backside attack of the leaving groups as nucleophilic reagents. Fracturing of old bonds and formation of new bonds simultaneously proceed. However, the stronger the nucleophilicity of the reagent in the $S_{N} 2$ reaction is, the higher the reaction velocity is. The nucleophilicity of $\mathrm{H}_{2} \mathrm{O}$ is smaller than that of methoxyl. Therefore, the reaction slowly occurs between DMP and $\mathrm{H}_{2} \mathrm{O}$ without catalyst, so slowly so that the reaction
TABLE 6: Results of the water content $\left(\mu \mathrm{g} \cdot \mathrm{g}^{-1}\right)$ in jet fuels under different conditions.

\begin{tabular}{lcccccc}
\hline \multicolumn{2}{c}{ Factors } & \multicolumn{5}{c}{ TMT } \\
$\begin{array}{l}\text { Temperature } \\
\left({ }^{\circ} \mathrm{C}\right)\end{array}$ & $\begin{array}{c}\text { Humidity } \\
(\%)\end{array}$ & $A$ & $B$ & $C$ & $\begin{array}{c}\text { Mean } \\
\text { value }\end{array}$ & $\begin{array}{c}\text { Karl } \\
\text { Fischer }\end{array}$ \\
\hline 0 & 60 & 30 & 36 & 39 & 35 & 31 \\
6 & 65 & 35 & 46 & 45 & 42 & 38 \\
9 & 71 & 52 & 45 & 53 & 50 & 51 \\
16 & 77 & 47 & 62 & 62 & 57 & 55 \\
20 & 80 & 57 & 54 & 63 & 58 & 60 \\
24 & 83 & 59 & 68 & 68 & 65 & 63 \\
31 & 85 & 70 & 75 & 83 & 76 & 69 \\
\hline
\end{tabular}

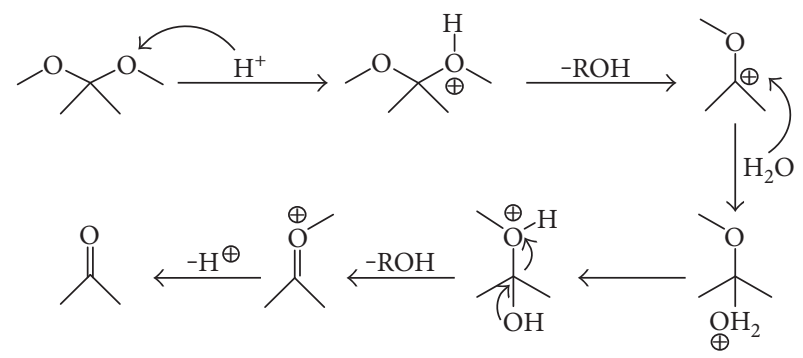

Figure 4: Proposed mechanism between DMP and $\mathrm{H}_{2} \mathrm{O}$.

cannot be noticed. Herein, we focus on acid as catalyst to accelerate the reaction and convert the reaction process from $\mathrm{S}_{\mathrm{N}} 2$ substitution into $\mathrm{S}_{\mathrm{N}} 1$ substitution. Protonation effect occurs in DMP because of the bond between the proton and methoxyl. As a result, methyl alcohol molecules were separated from DMP and formed carbocations. After a methoxyl was lost, steric hindrance was reduced, so it would be easily attacked by the nucleophilic reagent. As the nucleophilic reagent, $\mathrm{H}_{2} \mathrm{O}$ was bonded with carbocations, then the 
second methyl alcohol molecule was separated from a center carbon atom, and formation of protonated acetone occurred. As heat was absorbed in this process, the protons were finally removed to form acetone, and the removed protons continued the catalytic reaction.

\section{Conclusion}

The determination results on $0.01 \%-0.2 \%(\mathrm{~m} / \mathrm{m})$ spiked water were highly accurate and good repeatable. The obtained results on trace water in jet fuel at different temperature and humidity conditions were in accordance with those obtained using the Karl Fischer method. The duration of the entire determination process was 3-5 min. The trace water in jet fuels could be accurately and rapidly determined by TMT. As a result, TMT was appropriate for the determination of trace water in jet fuels.

\section{Conflicts of Interest}

The authors declare that they have no conflicts of interest.

\section{Acknowledgments}

This work was subsidized the fund from the National Natural Science Foundation of China (Grant 51575525), the Natural Science Foundation of Jiangsu Province (Grants BK20151157, BK20150166, and BK20161188), Natural Science foundation of the Anhui Higher Education Institutions of China (Grant KJ2017A400), and the project was supported by the Tribology Science Fund of State Key Laboratory of Tribology (Grant SKLTKF14B10).

\section{References}

[1] K. Chen, H. Xiang, T. Sun, and D. M. Dian, "Research on the icing behavior of water in the jet fuel," Petrochemical Industry Applicant, vol. 33, no. 12, pp. 1-5, 2014.

[2] N. Wu, J. Q. Hu, and S. Z. Yang, "Study on change rule of dissolved water content in jet fuels," Petroleum Processing and Petrochemicals, vol. 41, no. 9, pp. 22-24, 2015.

[3] X. G. Chen, X. X. Tian, and Y. Yang, "Research of on-line monitoring of trace moisture content in transformer oil," Journal of Southwest University (Natural Science Edition), vol. 31, no. 1, pp. 1-4, 2009.

[4] C. Zhang, X. Chen, D. J. Webb, and G.-D. Peng, "Water detection in jet fuel using a polymer optical fibre Bragg grating," 20th International Conference on Optical Fibre Sensors, vol. 75, no. 03, pp. 81-84, 2009.

[5] G. H. Nicolta, I. H. Daniel, and I. Heinz-dieter, "Water content of natural cyclodextrins and their essential oil complexes: a comparative study between Karl Fischer titration and thermal methods," Food Chemistry, vol. 132, no. 4, pp. 17411748, 2012.

[6] F. M. Najib, S. Zewar, and A. M. Abdulla, "A new sensor for thermometric titrations," Talanta, vol. 71, no. 1, pp. 141148, 2007.

[7] P. E. G. Jean and M. R. José, "Isothermal titration calorimetry: a thermodynamic interpretation of measurements," Chemical Thermodynamics, vol. 55, pp. 193-202, 2012.
[8] G. Burgot, F. Auffret, and J. L. Burgot, "Determination of acetaminophen by thermometric titrimetry," Analytica Chimica Acta, vol. 343, no. 1-2, pp. 125-728, 1997.

[9] S. U. Huan, W. A. Hart, and G. O. Yan, "Determination of sulfate ion FGD equipment from fossil fuel power plants by thermometric titration," Thermal Power Generation, vol. 44, no. 4, pp. 116-119, 2015.

[10] T. K. Smith, "Analysis of FFA in edible oils by catalyzed end-point thermometric titrimetry (CETT)," Journal of the American Oil Chemists' Society, vol. 80, no. 1, pp. 21-24, 2003.

[11] S. Z. Yang, J. Q. Hu, and L. Guo, "Rapid determination for acidity of aviation oils by thermometric titration," Acta Petrolerei Sinica (Petroleum Processing Section), vol. 30, no. 1, pp. 71-75, 2014.

[12] E. J. Greenhow, "Catalytic thermometric Titrimetry," Chemical Reviews, vol. 77, no. 6, pp. 835-854, 1977. 

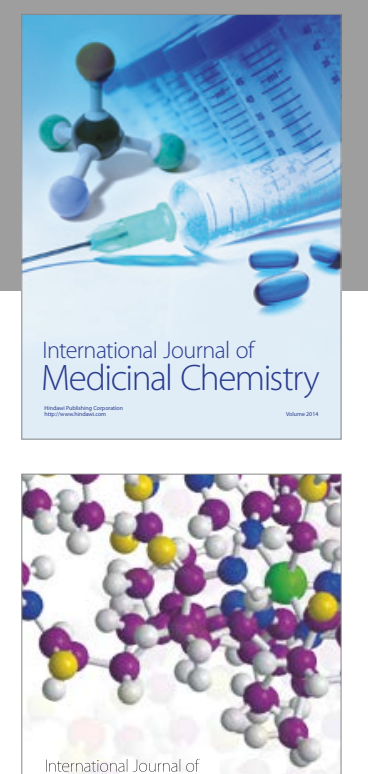

Carbohydrate Chemistry

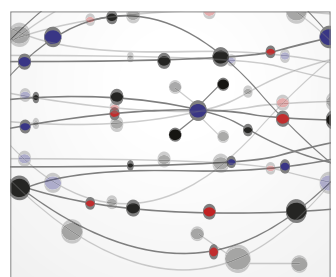

The Scientific World Journal
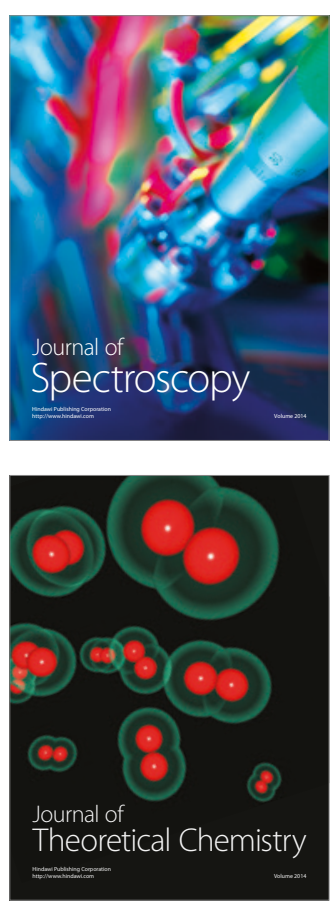
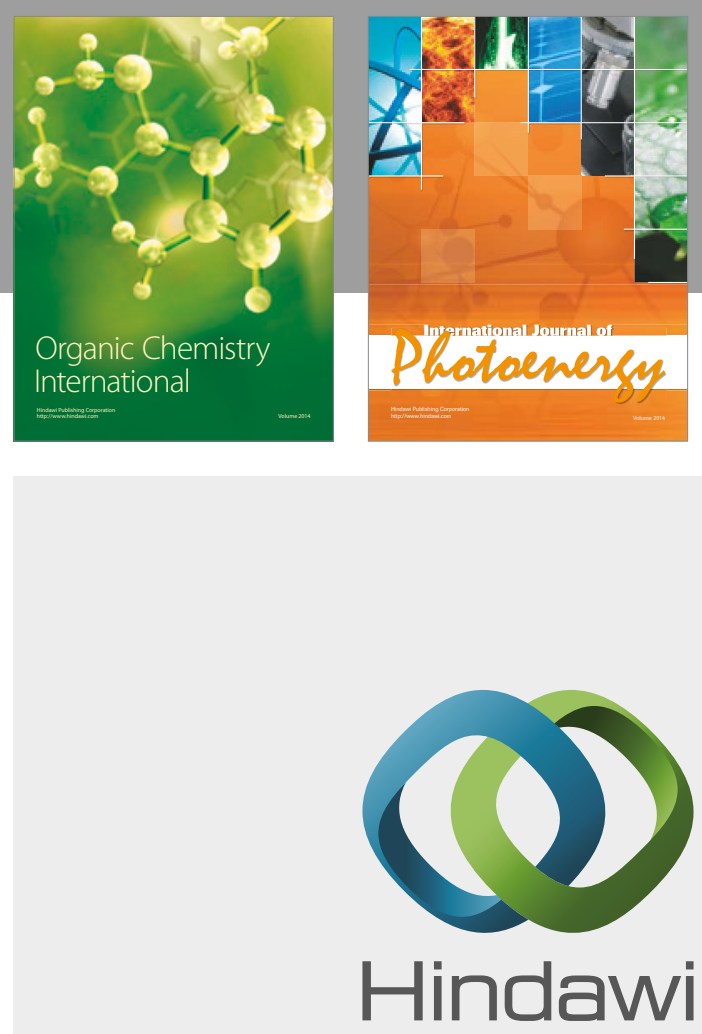

Submit your manuscripts at

https://www.hindawi.com

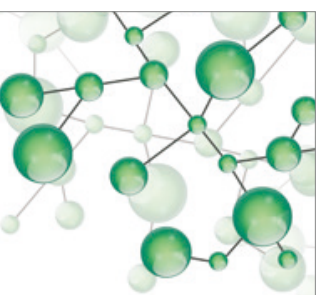

International Journal of

Inorganic Chemistry

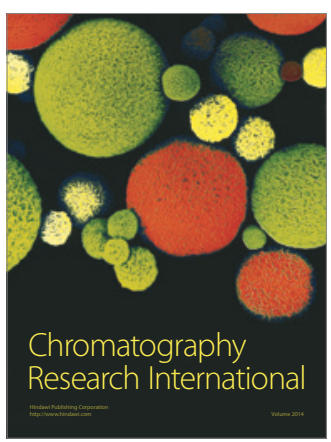

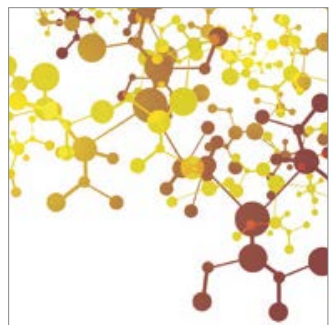

Applied Chemistry
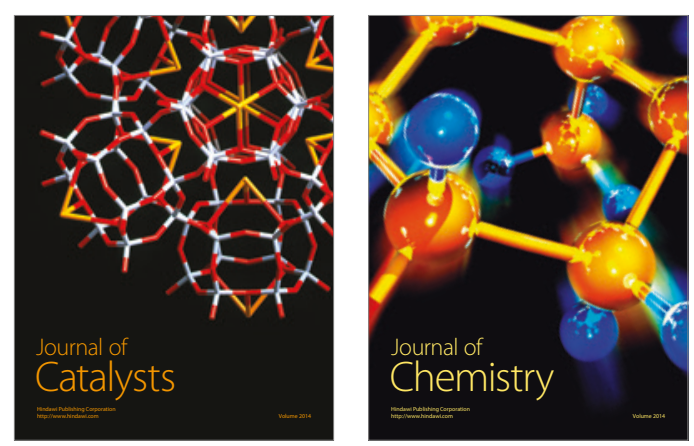
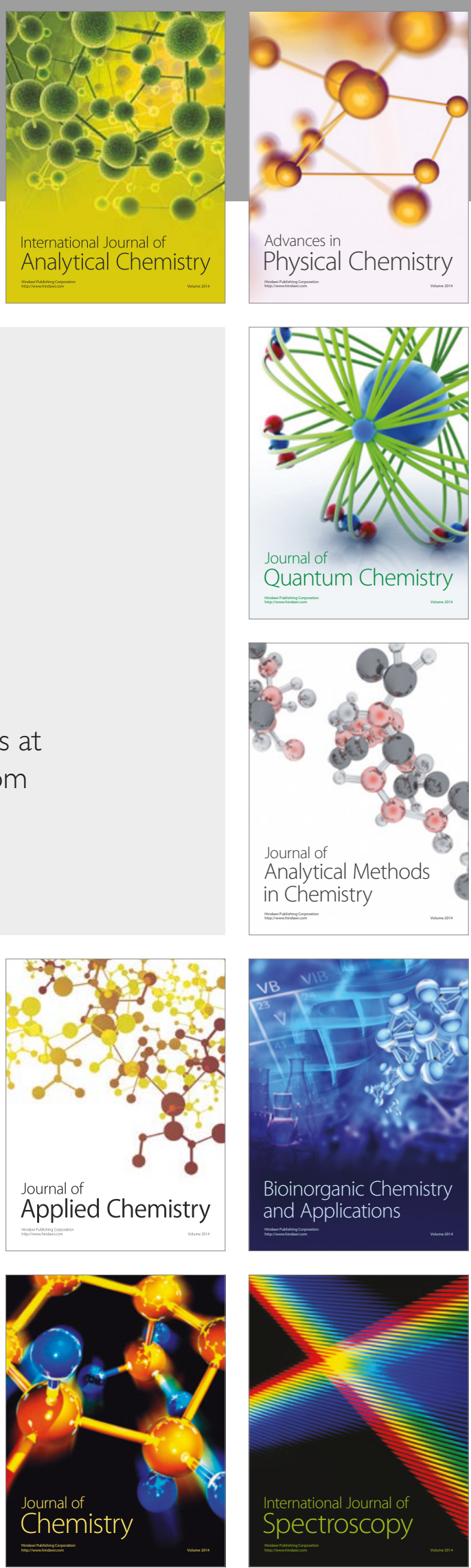